\title{
Percutaneous Cannulation for Extracorporeal Life Support in Severely and Morbidly Obese Patients
}

Journal of Intensive Care Medicine I-8

(C) The Author(s) 2018 Article reuse guidelines: sagepub.com/journals-permissions DOI: I0.II77/08850666I880I547 journals.sagepub.com/home/jic (S)AGE

\author{
Andreas Keyser, $\mathbf{M D}^{\prime}{ }^{\oplus}$, Alois Philipp', Florian Zeman, $\mathbf{M S c}^{2}$, \\ Matthias Lubnow, MD, PhD ${ }^{3}$, Dirk Lunz, MD ${ }^{4}$, \\ Markus Zimmermann, MD, $\mathrm{PhD}^{4}$, and Christof Schmid, MD, PhD ${ }^{1}$
}

\begin{abstract}
Background: Extracorporeal life support systems are well-established devices for treating patients with acute cardiopulmonary failure. Severe or morbid obesity may result in complications such as limb ischemia, bleeding, unsuccessful cannulation, or infection at the cannulation sites. This article reports on our experience with cannulation and associated complications in severely and morbidly obese patients. Methods: Between January 2006 and September 2016, I53 severely or morbidly obese patients with a body mass index $>35 \mathrm{~kg} / \mathrm{m}^{2}$ were cannulated percutaneously for extracorporeal life support at our center. Among those, II 5 patients were treated with venovenous extracorporeal membrane oxygenation (VV ECMO) for acute lung failure and 38 patients with venoarterial extracorporeal membrane oxygenation (VA ECMO) for cardiogenic shock. Complications related to percutaneous access and long-term follow-up were analyzed retrospectively. Primary focus was on the success of cannulation, outcome, thrombosis, bleeding, limb ischemia, and infection at the cannulation site. Normal-weight patients receiving extracorporeal life support served as control. Results: Percutaneous cannulation was successfully performed in all patients. Eighty-five (74\%) patients were weaned from VV ECMO and 20 (52\%) patients were weaned from VA ECMO. Limb ischemia requiring surgical intervention occurred in $5(3 \%)$ patients, bleeding in $7(5 \%)$ patients, and wound infection in $3(2 \%)$ patients. In all other patients, decannulation was uneventful. These data as well as the long-term survival rates were comparable to those of normalweight patients $(P>.05)$. Conclusion: Percutaneous vessel cannulation for extracorporeal life support systems is generally feasible. Therefore, percutaneous cannulation may well be performed in severely and morbidly obese patients. Patient outcome rather depends on appropriate support than on anatomy.
\end{abstract}

\section{Keywords}

extracorporeal life support, extracorporeal membrane oxygenation, percutaneous cannulation, obesity

\section{Background}

Extracorporeal life support systems are well-established devices for treating patients with acute cardiopulmonary failure in specialized facilities. ${ }^{1}$ Improvements in cannulation techniques and support devices enable the initiation of extracorporeal cardiorespiratory support virtually everywhere. The devices are implantable even far from cardiosurgical units in emergency departments or nonsurgical intensive care wards and as part of rescue conditions outside hospitals. The indications for venovenous, venoarterial, or pumpless extracorporeal assists are well defined and are subject to recommendations of the Extracorporeal Life-Support Organization.

Patient survival depends on the selection of the appropriate support device and its management. ${ }^{1,2}$ Severe obesity is often considered a contraindication to extracorporeal life support because of the considerable technical challenge during percutaneous cannulation. ${ }^{3}$ Apart from the most frequent complications, leg ischemia and bleeding, the most feared complications are unsuccessful cannulation and infections at the cannulation site. ${ }^{2}$ The article reports

\footnotetext{
' Department of Cardiothoracic Surgery, University Medical Center Regensburg, Regensburg, Germany

${ }^{2}$ Center for Clinical Studies, University Medical Center Regensburg, Regensburg, Germany

${ }^{3}$ Department of Cardiology, University Medical Center Regensburg, Regensburg, Germany

${ }^{4}$ Department of Anesthesiology, University Medical Center Regensburg, Regensburg, Germany
}

Received May II, 2018. Received revised August 27, 2018. Accepted August 29, 2018.

\section{Corresponding Author:}

Andreas Keyser, Department of Cardiothoracic Surgery, University Medical Center Regensburg, Franz-Josef-Strauss-Allee II, Regensburg 93053, Germany. Email: andreas.keyser@ukr.de 


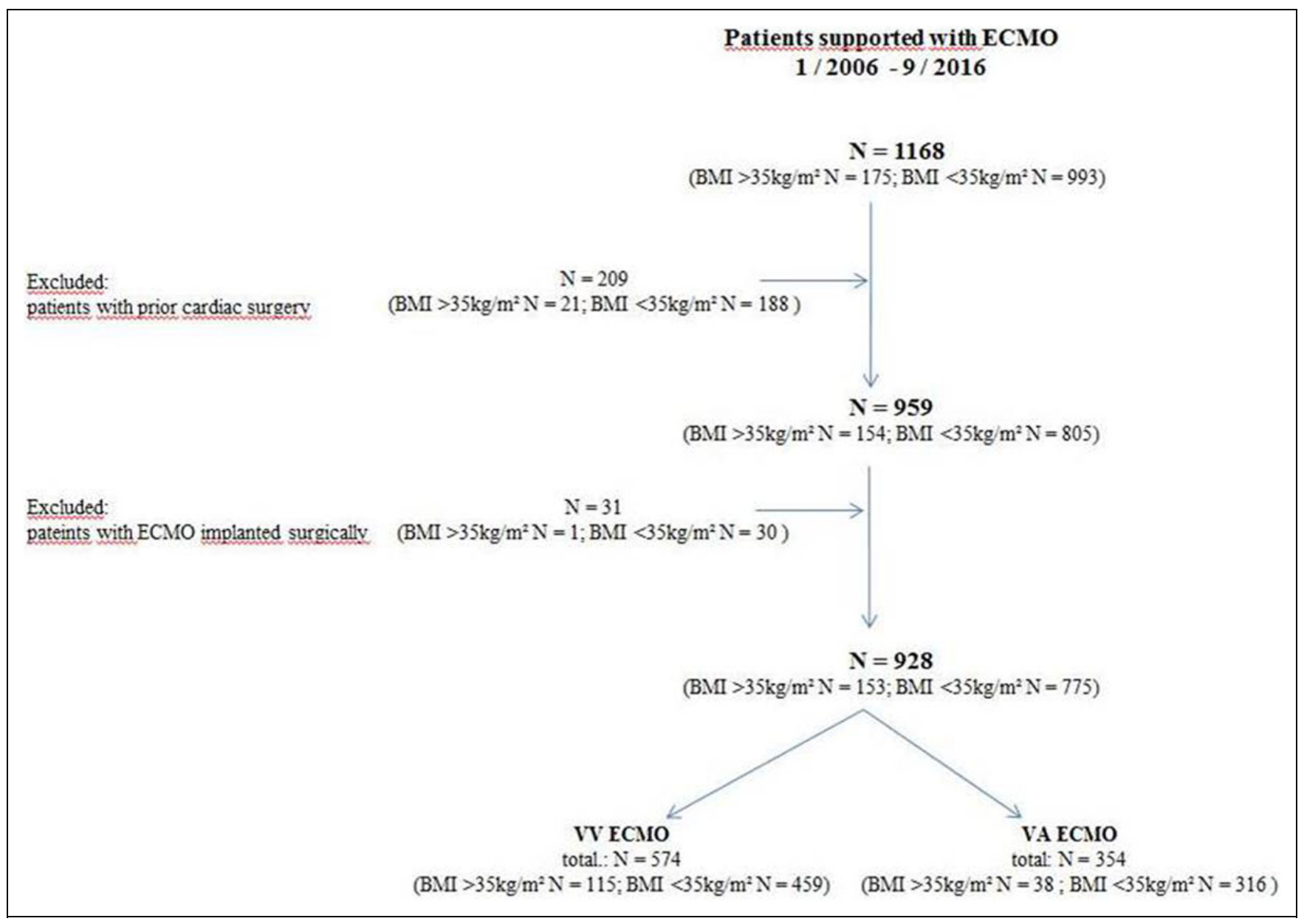

Figure I. Patient selection diagram. BMI indicates body mass index; VA ECMO, venoarterial extracorporeal life-support; VV ECMO, venovenous extracorporeal life-support.

on our experience with percutaneous cannulation for extracorporeal life support systems and associated complications in severely and morbidly obese patients without prior cardiac surgery.

\section{Methods}

\section{Study Population}

Between January 2006 and September 2016, 1168 extracorporeal life support systems were implanted. All data were prospectively saved in a database on an access-controlled personal computer. Patients who had undergone cardiac surgery prior to extracorporeal life support were excluded. A surgically implanted cannula for extracorporeal support was also considered an exclusion criterion. The patients were retrospectively analyzed according to the extracorporeal support required. Severe obesity was defined as a body mass index (BMI) of $>35 \mathrm{~kg} / \mathrm{m}^{2}$ according to World Health Organization definition (Figure 1).

\section{Cannulation}

Prior to cannulation, the access vessels were evaluated by means of duplex ultrasound, unless resuscitation was ongoing.
After puncture of the vessel and insertion of a guidewire, a dose of $5000 \mathrm{IU}$ of heparin was injected. The vascular access was gradually dilated (Percutaneous Insertion Kit; Maquet Cardiopulmonary AG, Rastatt, Germany) up to the size of the chosen cannula, facilitating cannula placement. Special equipment may be required for percutaneous implantation such as special guidewires (ie Amplatz Super Stiff, Boston Scientific, Massachusetts). Arterial cannulas sized $15 \mathrm{~F}$ or $17 \mathrm{~F}$ (length $23 \mathrm{~cm}$ ) were inserted, and venous cannulation was performed with cannulas of $21 \mathrm{~F}$ (length $38 \mathrm{~cm}$ ). Distal leg perfusion has been used as a standard procedure since 2014 .

For the cannulation using a dual lumen cannula (Avalon cannula; Maquet Cardiopulmonary AG, Rastatt, Germany), duplex sonography of the right jugular vein was deemed mandatory for choosing the correct anatomic localization and size and for excluding thrombotic occlusion. The cannula was percutaneously implanted according to the Seldinger technique, generally supported by transesophageal echocardiography and, in rare cases, by fluoroscopy. Cannulation of the subclavian vein as a return vessel access (size 15F-17F) for venovenous extracorporeal membrane oxygenation (VV ECMO) was also supported by transesophageal echocardiography. 


\section{Support and Complications}

On ECMO support, partial thromboplastin time (PTT) and antithrombin III (AT III) were monitored to ensure appropriate anticoagulation with heparin. If necessary, AT III was substituted to achieve a target value of $\geq 50 \%$. Administration of heparin was stopped 2 hours prior to decannulation. In general, decannulation was conducted by simply pulling out the cannula. The arterial cannula was removed first to maintain the possibility of infusing the volume via the venous cannula. After manual compression of the arterial puncture site, compression was continued by applying a Safeguard system (Pressure Assisted Device; Maquet Cardiopulmonary AG, Rastatt, Germany) for 24 hours.

Weaning and survival were analyzed as well as the type and incidence of complications related to the percutaneous access to femoral vessels and the jugular or subclavian vein. Implantations performed outside our center were also considered. The primary end point was on the success of cannulation. Bleeding at the cannulation site was defined by the requirement of the transfusion of packed red blood cells, the hemoglobin target for transfusion being $\geq 9 \mathrm{~g} / \mathrm{dL}$ at our institution. Limb ischemia requiring surgical treatment and infection at the cannulation site requiring surgical intervention were considered as complications.

\section{Statistics}

Normal distributed variables were summarized as mean values and standard deviation (SD), whereas skewed variables were presented as median (interquartile range [IQR]). Categorical data were summarized as frequency counts and percentages. Continuous variables were compared using the unpaired $t$ test for normal distributed variables and the nonparametric MannWhitney $U$-test for nonnormally distributed variables. Categorical variables were compared with a Pearson $\chi^{2}$ test of independence. Overall survivals (OS) were presented using Kaplan-Meier survival curves, and differences between BMI groups were compared to the nonparametric log-rank test. All tests were 2 -sided, and values of $P<.05$ indicated a significant difference. All analyses were performed using R (version 3.3.3).

\section{Results}

\section{Patient Groups}

A total of 928 patients had received percutaneous cannulation for extracorporeal membrane oxygenation. Among those, 115 obese patients were supported with VV ECMO for acute respiratory failure, and 38 obese patients were supported with venoarterial ECMO (VA ECMO) after cardiogenic shock or resuscitation. The latter group included 5 patients with severe hypercapnia who had undergone arteriovenous extracorporeal carbon dioxide removal (AV ECCO2R). The AV ECCO2R patients were included in the VA ECMO group because they had also required arterial and venous cannula insertion.

\section{Demographic Data}

Severe or morbid obesity was found in $153(16.5 \%)$ of 928 patients with either isolated pulmonary or combined cardiopulmonary failure who had received cannulation for extracorporeal life support systems. The 38 obese patients ( 27 men and 11 women) who had received a VA ECMO because of cardiogenic shock and/or resuscitation had a mean age of $56.8 \pm 9.8$ years and a mean BMI of $42.3 \pm 12.4 \mathrm{~kg} / \mathrm{m}^{2}$ (range: $35-69.2 \mathrm{~kg} / \mathrm{m}^{2}$ ). A VV ECMO had been placed in 115 patients ( 76 men and 39 women) who had a mean age of $53.8 \pm 12.4$ years and a mean BMI of $43.9 \pm 10.1 \mathrm{~kg} / \mathrm{m}^{2}$ (range: $35-84.5 \mathrm{~kg} / \mathrm{m}^{2}$ ). The obese VV ECMO patients were significantly older than the normalweight patients $(53.83 \pm 12.35$ vs $48.42 \pm 17.5, P<.05)$ and had more often been referred to our center from a noncardiosurgical center (62 [54\%] vs 197 [43\%], $P<.05)$. The sequential organ failure assessment (SOFA) score appeared to be comparable between severely or morbidly obese patients and normal-weight patients in both the VV and the VA ECMO group (VV ECMO $12.32 \pm 3.22$ vs $11.35 \pm 3.94, P>.05$, VA ECMO $13.09 \pm 2.98$ vs $11.82 \pm 3.15, P<.05)$.

However, renal failure prior to VA ECMO support was apparent in 15 (39\%) obese patients when compared to 50 $(16 \%)$ normal-weight patients, which had statistical relevance $(P<.05)$. Multiple organ failure was seen in $23(61 \%)$ obese patients when compared to $129(42 \%)$ normal-weight patients. This finding was also statistically significant $(P<.05)$. In the VV ECMO group, multiple organ failure was observed in 56 $(49 \%)$ obese patients and in $183(40 \%)$ normal-weight patients $(P<.05)$. Demographic data are shown in Table 1 for VA ECMO patients and in Table 2 for VV ECMO patients.

\section{Cannulation}

Cannulation was successfully performed in all 153 patients. In the VA ECMO group, the return cannula could always be implanted into a femoral artery. Drainage cannula access was gained via the femoral vein in 34 patients and via the jugular vein in 4 patients. A VA ECMO was placed in a referral hospital in 12 patients, and 2 VA ECMOs were implanted by our mobile ECMO team during extended cardiopulmonary resuscitation outside a hospital. Obese patients had more often received a jugular vein access than a femoral access for return cannula than normal-weight patients $(P<.05)$.

A femoro-jugular access was chosen in $70(60.9 \%)$ obese VV ECMO patients, a femoro-femoral access in 7 (6.1\%), and a femoro-subclavian access in $12(10.4 \%)$ patients. In the latter group, the subclavian vein served as a return vessel (see Table 3).Decannulation was event free without any surgical standby. In $26(22.6 \%)$ patients, the dual lumen catheter technique via the jugular vein (Avalon cannula) was applied. Obese patients were more often treated with the Avalon cannula than normalweight patients (26 [23\%] patients vs 62 [14\%] patients, $P<$ $.05)$, that is, the jugular access was used more often in obese patients $(P<.05)$. 
Table I. Demographic data of patients supported with VA ECMO.

\begin{tabular}{|c|c|c|c|}
\hline \multirow[b]{2}{*}{ Variable } & \multicolumn{3}{|c|}{ VA ECMO } \\
\hline & $\mathrm{BMI}>35 \mathrm{~kg} / \mathrm{m}^{2}$ & $\mathrm{BMI}<35 \mathrm{~kg} / \mathrm{m}^{2}$ & $\begin{array}{c}P \\
\text { Value }\end{array}$ \\
\hline Age, years & $56.84 \pm 9.82$ & $56.00 \pm 14.46$ & $>.05$ \\
\hline \multicolumn{4}{|l|}{ Gender } \\
\hline Male & 27 (7I\%) & $233(74 \%)$ & $>.05$ \\
\hline Female & II (29\%) & $83(26 \%)$ & \\
\hline Height, cm & $170.63 \pm 14.60$ & $172.13 \pm 7.20$ & $>.05$ \\
\hline Weight, kg & $|20.7| \pm 24.97$ & $77.96 \pm 12.22$ & $<.05$ \\
\hline $\mathrm{BMI}$ & $42.29 \pm 12.44$ & $26.25 \pm 3.31$ & $<.05$ \\
\hline \multicolumn{4}{|l|}{$\begin{array}{l}\text { Resuscitation prior to } \\
\text { support }\end{array}$} \\
\hline Yes & $29(76 \%)$ & $263(83 \%)$ & $>.05$ \\
\hline No & $9(24 \%)$ & $53(17 \%)$ & \\
\hline \multicolumn{4}{|l|}{ Mode of resuscitation } \\
\hline None & $9(24 \%)$ & $56(18 \%)$ & $>.05$ \\
\hline Mediction only & $2(5 \%)$ & $29(9 \%)$ & \\
\hline Mechanical & $24(63 \%)$ & $199(63 \%)$ & \\
\hline $\begin{array}{l}\text { Mechanical assisted } \\
\text { (LUKAS) }\end{array}$ & $3(8 \%)$ & $32(10 \%)$ & \\
\hline \multicolumn{4}{|l|}{ Referral } \\
\hline Own hospital & $19(50 \%)$ & 155 (49\%) & $>.05$ \\
\hline $\begin{array}{l}\text { Distant } \\
\text { noncardiosurgical } \\
\text { hospital }\end{array}$ & $17(45 \%)$ & 142 (45\%) & \\
\hline Out of hospital & $2(5 \%)$ & $19(6 \%)$ & \\
\hline \multicolumn{4}{|l|}{ ECMO transportation } \\
\hline None & $24(63 \%)$ & $192(61 \%)$ & $>.05$ \\
\hline Ambulance car & $10(26 \%)$ & $57(18 \%)$ & \\
\hline Helicopter & $4(\mathrm{II} \%)$ & $67(21 \%)$ & \\
\hline SOFA score & $13.09 \pm 2.98$ & $11.82 \pm 3.15$ & $>.05$ \\
\hline
\end{tabular}

Abbreviations: SOFA, sequential organ failure assessment; VV ECMO, venovenous extracorporeal membrane oxygenation; BMI; body mass index.

It is noteworthy that $61(53 \%)$ of the patients had the VV ECMO implanted by our mobile ECMO team in another institute and were afterward transferred to our hospital by helicopter or ambulance (Table 2).

\section{Support and Complications}

Median support interval was 5 days (IQR: 2-7, range: 0-20 days) in the VA ECMO group and 9 days (IQR: 6-14, range: 0-63 days) in the VV ECMO group. No statistical difference to normal-weight patients could be found, neither in the VA ECMO support (3 days; IQR: 1.75-6, range: 0-85 days) nor in the VV ECMO group (8 days; IQR: 5-14, range: 0-94 days, $P>.05)$. Twenty $(52 \%)$ obese patients treated with VA ECMO - as well as all obese patients treated with AV ECCO2R - were successfully weaned from the system. Six patients died during follow-up, that is, overall 14 (37\%) patients survived and could be discharged from hospital. Weaning was successful in 85 of the obese patients treated with VV ECMO (74\%), of whom 76 (66\%) patients survived and could be discharged from the hospital.
Table 2. Demographic data of patients supported with VV ECMO.

\begin{tabular}{|c|c|c|c|}
\hline \multirow[b]{2}{*}{ Variable } & \multicolumn{3}{|c|}{ VV ECMO } \\
\hline & $\mathrm{BMI}>35 \mathrm{~kg} / \mathrm{m}^{2}$ & $\mathrm{BMI}<35 \mathrm{~kg} / \mathrm{m}^{2}$ & $P$ Value \\
\hline Age, years & $53.83 \pm 12.35$ & $48.42 \pm 17.50$ & $<.05$ \\
\hline \multicolumn{4}{|l|}{ Gender } \\
\hline Male & $76(66 \%)$ & $315(69 \%)$ & $>.05$ \\
\hline Female & $39(34 \%)$ & | 44 (3|\%) & \\
\hline Height, cm & $170.74 \pm 7.29$ & $171.10 \pm 14.79$ & $>.05$ \\
\hline Weight, kg & $127.50 \pm 26.98$ & $78.45 \pm 16.50$ & $<.05$ \\
\hline $\mathrm{BMI}$ & $43.92 \pm 10.15$ & $26.42 \pm 4.10$ & $<.05$ \\
\hline \multicolumn{4}{|l|}{$\begin{array}{l}\text { Resuscitation prior to } \\
\text { support }\end{array}$} \\
\hline Yes & 93 (8I\%) & $382(83 \%)$ & $>.05$ \\
\hline No & 22 (19\%) & 77 (I7\%) & \\
\hline \multicolumn{4}{|l|}{ Mode of resuscitation } \\
\hline None & 103 (90\%) & 402 (88\%) & $>.05$ \\
\hline Medication only & II (10\%) & 49 (1 I\%) & \\
\hline Mechanical & I (I\%) & $8(2 \%)$ & \\
\hline \multicolumn{4}{|l|}{$\begin{array}{l}\text { Mechanical assisted } \\
\text { (LUKAS) }\end{array}$} \\
\hline \multicolumn{4}{|l|}{ Referral } \\
\hline Own hospital & $53(46 \%)$ & $262(57 \%)$ & $<.05$ \\
\hline $\begin{array}{l}\text { Distant } \\
\text { noncardiosurgical } \\
\text { hospital }\end{array}$ & $62(54 \%)$ & 197 (43\%) & \\
\hline \multicolumn{4}{|l|}{ Out of hospital } \\
\hline \multicolumn{4}{|l|}{ ECMO transportation } \\
\hline None & 54 (47\%) & $26 \mid(57 \%)$ & $<.05$ \\
\hline Ambulance car & $26(23 \%)$ & $62(14 \%)$ & \\
\hline Helicopter & $35(30 \%)$ & $136(30 \%)$ & \\
\hline SOFA score & $12.32 \pm 3.22$ & $11.35 \pm 3.94$ & $>.05$ \\
\hline
\end{tabular}

Abbreviations: SOFA, sequential organ failure assessment; VV ECMO, venovenous extracorporeal membrane oxygenation; BMI; body mass index.

No significant difference in OS was found between severely and morbidly obese VV or VA ECMO patients and normalweight patients $(P>.05$; see Figures 2 and 3$)$.

Inappropriate cannulation such as malposition and dislocation required relocation of the return cannula in $4(10 \%)$ obese patients supported with VA ECMO and in $6(5 \%)$ patients supported with VV ECMO. In normal-weight patients, relocation of the return cannula was necessary in $41(13 \%)$ patients supported with VA ECMO and in $15(3 \%)$ patients supported with VV ECMO. No statistically significant difference could be found between obese and normal-weight patients in either ECMO group. When compared to normal-weight patients, severely or morbidly obese patients had significantly more often thrombotic occlusions of the VV ECMO system (48 [42\%] obese patients and 130 [28\%] normal-weight patients, $P<.05$ ).

Obese patients had a similar risk of requiring transfusion of packed red blood cells than normal-weight patients (0; IQR: $0-2$, range: $0-14$ vs 0 and IQR: $0-4$; range: $0-35, P>.05$ ). Also, obese patients did not require more thrombocyte concentrates than normal-weight patients ( 0 ; IQR: $0-0$, range: $0-4$ vs 0 ; IQR: $0-0$, range: $0-13, P>.05)$. No differences in weaning, survival, 
Table 3. Cannulation.

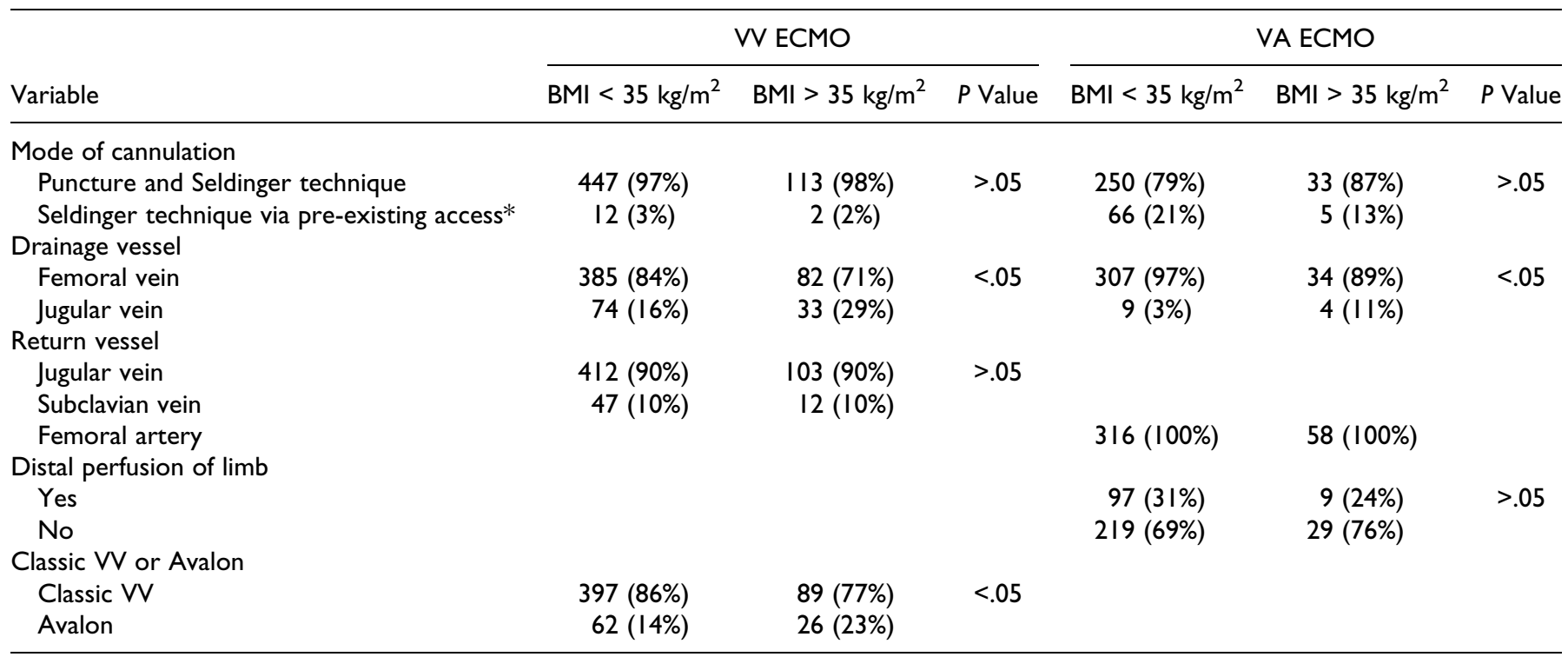

Abbreviations: BMI, body mass index; VV ECMO, venovenous extracorporeal membrane oxygenation; VA ECMO, venoarterial extracorporeal membrane oxygenation.

*arterial line or intra-aortic blood pump.

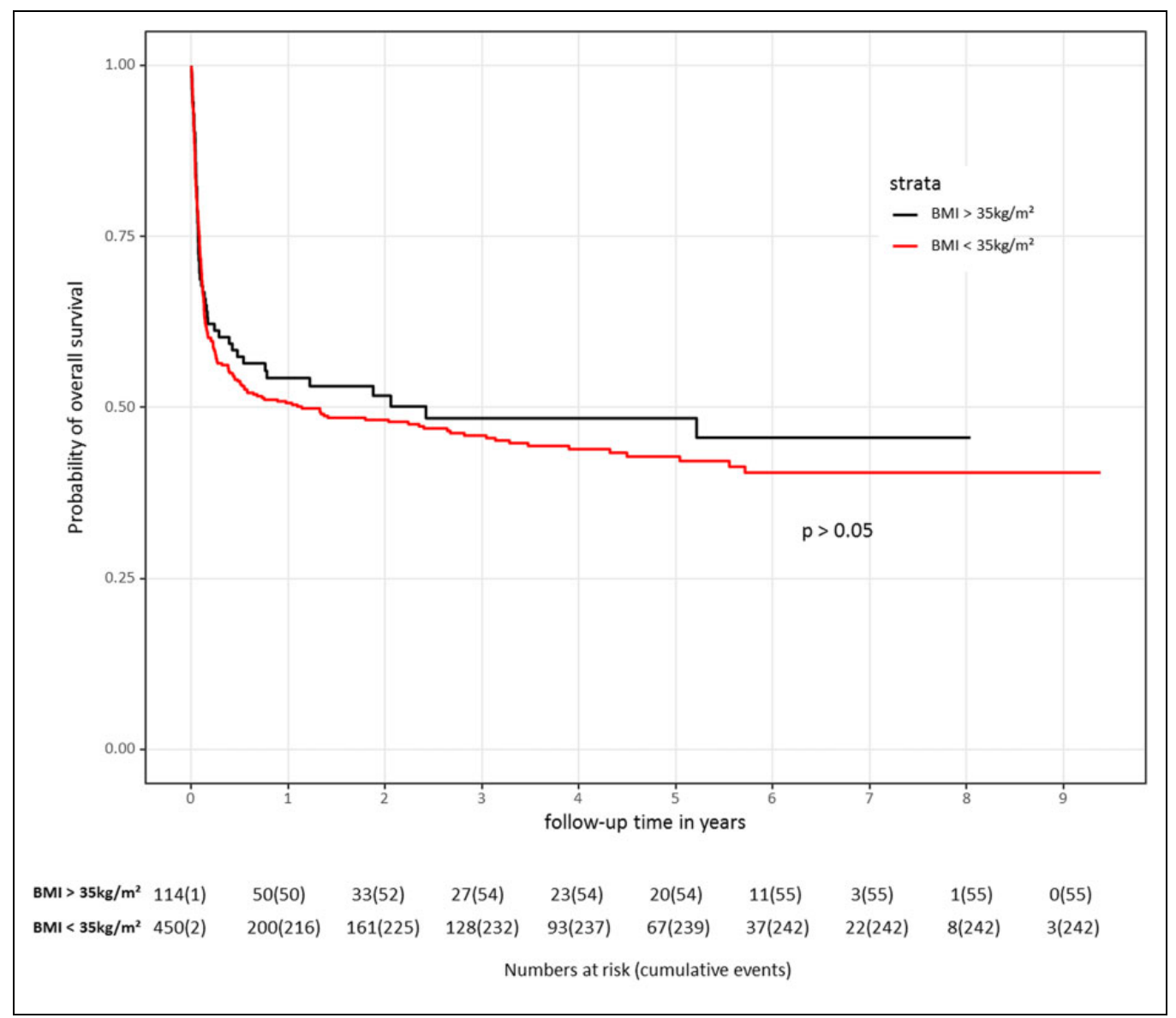

Figure 2. Long-term follow-up of VV ECMO patients. Overall survival curves for patients treated with venovenous extracorporeal life-support (VV ECMO) based on Kaplan-Meier produc. BMI indicates body mass index. 


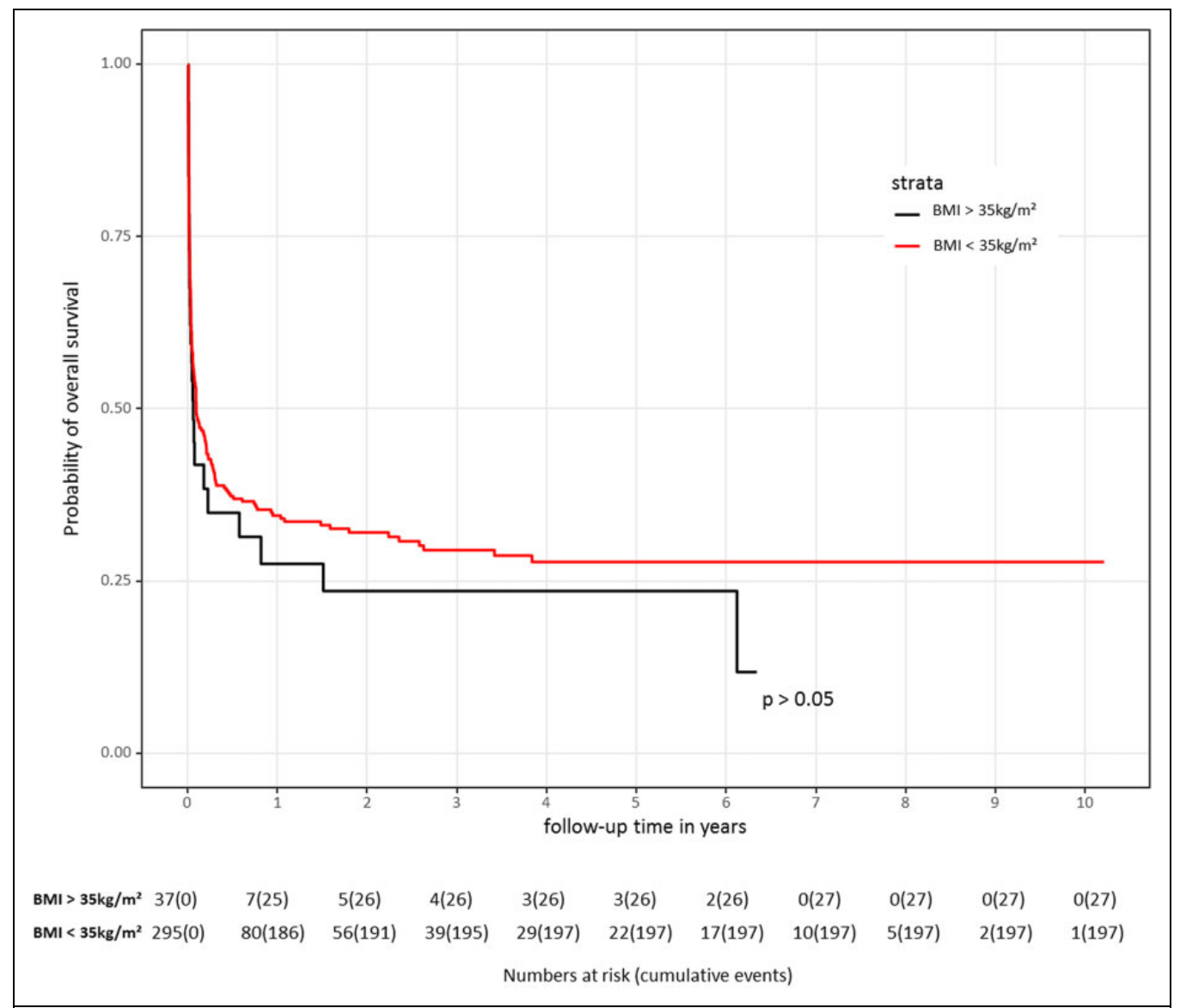

Figure 3. Long-term follow-up of VA ECMO patients. Overall survival curves for patients treated with venoarterial extracorporeal life-support (VA ECMO) based on Kaplan-Meier product. BMI indicates body mass index.

cause of death, technical problems, and complications were found between obese and normal-weight patients.

Due to bleeding, $6(5 \%)$ patients required a blood transfusion during implantation of VV ECMO, and 1 (3\%) obese patient in the VA ECMO group required surgical revision due to bleeding at the cannulation site. Bleeding at the cannulation site occurred in $31(10 \%)$ normal-weight patients in the VA ECMO group and in 21 (6\%) patients in the VV ECMO group. No significant difference in normal-weight patients was found $(P>.05)$.

Surgical intervention for limb ischemia was required in 5 (3\%) obese patients including 1 patient of the VV ECMO group. Limb ischemia was observed in $44(14 \%)$ normalweight patients on VA ECMO support and in $21(7 \%)$ patients after decannulation. In the VV ECMO group, 4 (1\%) normalweight patients had developed limb ischemia due to bleeding at the cannulation site and compression of the adjacent femoral artery.

Infection of the cannulation site was observed in $3(2 \%)$ obese patients and $5(1 \%)$ normal-weight patients. All patients were treated conservatively. No statistically significant difference has been found between obese and normal-weight patients concerning limb ischemia or infection (both $P>.05$ ).

\section{Discussion}

As a result of numerous improvements in experience and technology, extracorporeal life support has become a widely accepted device. ${ }^{1}$ By now, the implantation numbers of extracorporeal life-support systems have increased, and hardly any obstacles exist neither with regard to the place of device implantation nor the duration of support. ${ }^{4}$ However, in severe obese patients, the cannulation procedure can be challenging. Apart from obvious cannulation obstacles, the risk of complications is immanent. Therefore, appropriate patient selection, a highly skilled team, and a sufficient training program are important to avoid severe complications.

The range of obesity in both our ECMO groups is distinct. The cutoff of the BMI of $35 \mathrm{~kg} / \mathrm{m}^{2}$ was chosen according to the definition of Sturm. ${ }^{5}$ A further splitting of patients was not considered sensible with respect to the well-known fact that the BMI is not necessarily a negative predictor for outcome in patients with critical illness. ${ }^{6,7}$ Similarly, such patients should not be denied extracorporeal life-support systems because of their BMI as peripheral cannulation is easy to conduct. ${ }^{3}$ Moreover, such supposedly difficult cannulations may also be easily accomplished outside a center providing the ECMO program. Interestingly, inappropriate cannulation was rarely observed in 
our group of severely and morbidly obese patients, of whom only 4 patients treated with VA ECMO and 6 patients treated with VV ECMO had required relocation of the cannula to the opposite site. Generally, obese patients received a jugular vein access more often than a femoral access compared to normalweight patients because the insertion of a venous drainage for VA ECMO or an AVALON cannula in VV ECMO seems to be easier in obese patients.

Obese patients treated with VV ECMO were significantly older than normal-weight patients treated with VV ECMO. Furthermore, obese patients were more often referred to our center from hospitals without a VV ECMO program than normal-weight patients. Nevertheless, we did not find any difference in the outcome between VA and VV ECMO. These finding shows that extracorporeal life-support systems may be used literally anywhere and that outcome is closely related to selecting and managing the appropriate support tool. ${ }^{1}$

Patient outcome is influenced by underlying disease. The best survival rates have been achieved in patients with less comorbidities. Risk factors negatively affecting survival were advanced age as well as renal and multiple organ failure. ${ }^{8}$ In our experience, the SOFA score appears to be comparable between severely or morbidly obese and normal-weight patients. Obese patients not only seem to have a history of renal failure more often than normal-weight patients but also have a significantly higher risk of multiple organ failure. In spite of higher incidences of comorbidities, obese and critically ill patients tend to have a markedly high survival benefit. ${ }^{9}$ In contrast to the study by Abhyankar et al, the present study on ECMO did not yield any difference in the outcome and followup between severely or morbidly obese and normal-weight patients. Swol et al analyzed the relationship between BMI and outcome of 89 surgical patients supported with VV ECMO. Forty-five patients had a BMI of $>30 \mathrm{~kg} / \mathrm{m}^{2}$. Although a cutoff BMI of $30.66 \mathrm{~kg} / \mathrm{m}^{2}$ corresponded to the desired sensitivity and specificity to predict mortality, the authors admitted that the BMI had a poor decision-making power concerning ECMO support. ${ }^{10}$ A comparable outcome of patients supported by VV ECMO was also published by Kon et al. Their retrospective review study of 18 obese patients with a BMI of $>40 \mathrm{~kg} / \mathrm{m}^{2}$ in comparison to 43 normal-weight patients showed a similar outcome as well as comparable preexisting comorbidities of patients requiring VV ECMO for acute respiratory distress syndrome. ${ }^{11}$ Our study did not focus on comorbidities present prior to ECMO support. We assumed a heterogeneous group of patients because a substantial number of patients had been referred to our center. Nevertheless, the outcome for obese patients was not worse than that for normal-weight patients.

Obese and normal-weight patients in the VV and VA ECMO groups showed comparable mean support times. We did not find a significant difference in the average time of ECMO support in comparison to the ELSO register (register of the Extracorporeal Life-Support Organization). ${ }^{12}$ About $50 \%$ of the patients supported with VA ECMO, roughly $75 \%$ of the patients supported with VV ECMO, and all patients supported with AV ECCO2R could successfully be weaned. These results were consistent with the ELSO register as well. ${ }^{12}$

Overweight patients tended to need more heparin to achieve the target PTT of 50 to 60 seconds. Nevertheless, obese patients were more likely to experience clotting of the VV ECMO system than normal-weight patients. The association between obesity and thrombosis is well known. Elevated expression of the prothrombotic molecules and increased platelet activation are substantial parts of the pathomechanism. ${ }^{13,14}$ Furthermore, there is a link between inflammation and thrombogenicity. ${ }^{15}$ It is noteworthy that a statistically significant difference in thrombogenecity was found in VV ECMO patients but not in VA ECMO patients. Thrombogenecity in ECMO featuring artificial surfaces remains the subject of further investigation.

The 2 most frequent complications of ECMO are bleeding and infection at the cannulation site. In our study, bleeding requiring the transfusion of packed red blood cells was rare and comparable between severely or morbidly obese and normal-weight patients. The incidence of bleeding was low for VV and VA ECMO patients. Overall, we observed only one infection at the cannulation site after surgical explantation of the VV ECMO. The rate of our complications was comparable with that of the ELSO registry. ${ }^{12}$ Therefore, we decannulate by simply pulling out the cannulas.

Another major complication of ECMO systems is associated limb ischemia due to cannulation. An incidence of $13 \%$ to $20 \%$ has been reported with up to $50 \%$ of patients requiring fasciotomy. ${ }^{1,12,16-18}$ The patient cohort of our study had a remarkably low incidence of limb ischemia that was even lower than that reported in the literature. ${ }^{1}$ Distal limb perfusion, an essential component of VA ECMO support, may substantially contribute to reducing limb ischemia.

Our study has several limitations. It was designed as a retrospective study and conducted at one single medical center. There may be selection bias as to who has been supported by ECMO, therefore limiting the generalization of the findings. Furthermore, the etiology of the patients' diseases and the different conditions leading to the indication for ECMO support were neither specified nor compared, which may have influenced outcome and survival. Nevertheless, this study presents a reasonable number of patients supported with either VV or VA ECMO. Many of the patients had been transferred to our center. The follow-up data present the long-term outcome of patients supported by ECMO.

\section{Conclusion}

It is challenging but feasible to cannulate overweight patients when conducted by experienced physicians, even out of hospital or at referral hospitals. The outcome of patients with cardiorespiratory impairment rather depends on the choice of the appropriate support device and its implementation than on the anatomy of the patient. Obesity should not be considered a contraindication for VV ECMO or VA ECMO support. 


\section{Authors' Note}

$\mathrm{AK}, \mathrm{AP}, \mathrm{FZ}, \mathrm{ML}, \mathrm{DL}, \mathrm{MZ}$, and CS designed the study and are responsible for finalizing the protocol, analysis, and completion of the final manuscript. AK and AP developed the search strategy in consultation with FZ. AK conceived the project, developed the protocol, and wrote and revised the manuscript. CS contributed to the writing of the manuscript. All authors critically revised the protocol and the final manuscript. All authors read and approved the final manuscript. The authors declare that all data supporting the findings of this study are available within the article and its additional files.

\section{Declaration of Conflicting Interests}

The author(s) declared no potential conflicts of interest with respect to the research, authorship, and/or publication of this article.

\section{Disclosure and declaration}

The study was approved by the local Ethics Committee (reference number 16-104-0188), who waived the need for individual consent because of the retrospective and noninterventional design of the project.

\section{Funding}

The author(s) disclosed receipt of the following financial support for the research, authorship, and/or publication of this article: The study was not founded, neither regarding the design of the study, nor with regard to the collection, analysis, and interpretation of data, or the writing of the manuscript. None of the authors has any financial relationship with a commercial entity that has an interest in the subject of the manuscript or other conflicts of interest.

\section{ORCID iD}

Andreas Keyser, MD (D) http://orcid.org/0000-0001-5922-5288

\section{References}

1. Schmid C, Phillipp A, Mueller T, Hilker M. Extracorporeal life support-systems, indications, and limitations. Thorac Cardiovasc Surg. 2009;57(8):449-454.

2. Ganslmeier P, Philipp A, Rupprecht L, et al. Percutaneous cannulation for extracorporeal life support. Thorac Cardiovasc Surg. 2011;59(2):103-107.

3. Al-Soufi S, Buscher H, Nguyen DN, Rycus P, Nair P. Lack of association between body weight and mortality in patients on veno-venous extracorporeal membrane oxygenation. Intensive Care Med. 2013;39(11):1995-2002.

4. Rush B, Wiskar K, Berger L, Griesdale D. Trends in extracorporeal membrane oxygenation for the treatment of acute respiratory distress syndrome in the United States. $J$ Intensive Care Med. 2017;32(9):535-539.

5. Sturm R. Increases in morbid obesity in the USA: 2000-2005. Public Health. 2007;121(7):492-496.

6. Wacharasint P, Boyd JH, Russell JA, Walley KR. One size does not fit all in severe infection: obesity alters outcome, susceptibility, treatment, and inflammatory response. Crit Care. 2013;17(3): R122.

7. Esper AM, Martin GS. The impact of comorbid conditions on critical illness. Crit Care Med. 2011;39(12):2728-2735.

8. Vincent JL, Moreno R, Takala J, et al. The SOFA (sepsis-related organ failure assessment) score to describe organ dysfunction/ failure. Intensive Care Med. 1996;22(7):707-710.

9. Abhyankar S, Leishear K, Callaghan FM, Demner-Fushman D, McDonald CJ. Lower short- and long-term mortality associated with overweight and obesity in a large cohort study of adult intensive care unit patients. Crit Care. 2012;16(6):R235.

10. Swol J, Buchwald D, Strauch JT, Schildhauer TA, U1l C. Effect of body mass index on the outcome of surgical patients receiving extracorporeal devices (VV ECMO, pECLA) for respiratory failure. Int J Artif Organs. 2017;40(3):102-108.

11. Kon ZN, Dahi S, Evans CF, et al. Class III Obesity is not a contraindication to venovenous extracorporeal membrane oxygenation support. Ann Thorac Surg. 2015;100(5):1855-1860.

12. ECLS Registry Report. (2017) International Summary. www.elso. org. Accessed July, 2018.

13. Samad F, Ruf W. Inflammation, obesity, and thrombosis. Blood. 2013;122(20):3415-3422.

14. Morange PE, Alessi MC. Thrombosis in central obesity and metabolic syndrome: mechanisms and epidemiology. Thromb Haemost. 2013;110(4):669-680.

15. Hechler B, Gachet C. purinergic receptors in thrombosis and inflammation. Arterioscler Thromb Vasc Biol. 2015;35(11): 2307-2315

16. Bisdas T, Beutel G, Warnecke G, et al. Vascular complications in patients undergoing femoral cannulation for extracorporeal membrane oxygenation support. Ann Thorac Surg. 2011;92(2): 626-631.

17. Russo CF, Cannata A, Vitali E, Lanfranconi M. Prevention of limb ischemia and edema during peripheral venoarterial extracorporeal membrane oxygenation in adults. J Card Surg. 2009;24(2); 185-187.

18. Lamb KM, Hirose H, Cavarocchi NC. Considerations for percutaneous cannulation for veno-arterial extracorporeal membrane oxygenation. J Card Surg. 2013;28(2):190-192. 